\title{
Singularities of Type-Q ABS Equations ${ }^{\star}$
}

James ATKINSON

School of Mathematics and Statistics, The University of Sydney, NSW 2006, Australia

E-mail: james.atkinson@sydney.edu.au

Received February 14, 2011, in final form July 13, 2011; Published online July 20, 2011

doi:10.3842/SIGMA.2011.073

\begin{abstract}
The type-Q equations lie on the top level of the hierarchy introduced by Adler, Bobenko and Suris (ABS) in their classification of discrete counterparts of KdV-type integrable partial differential equations. We ask what singularities are possible in the solutions of these equations, and examine the relationship between the singularities and the principal integrability feature of multidimensional consistency. These questions are considered in the global setting and therefore extend previous considerations of singularities which have been local. What emerges are some simple geometric criteria that determine the allowed singularities, and the interesting discovery that generically the presence of singularities leads to a breakdown in the global consistency of such systems despite their local consistency property. This failure to be globally consistent is quantified by introducing a natural notion of monodromy for isolated singularities.
\end{abstract}

Key words: singularities; integrable systems; difference equations; multidimensional consistency

2010 Mathematics Subject Classification: 35Q58

\section{Introduction}

The motivation for the present work comes from a desire to understand non-generic behaviour of the multidimensionally consistent systems listed in [1] (cf. [2, 3]) which can arise due to the presence of singularities. Singularities in solutions of these systems have been considered previously. A class of solutions which are singular everywhere have been known since [4], their main interesting feature being a failure to significantly alter after application of the Bäcklund transformation. Also recall that a local consideration of singularities was found in [5] to underlie the classification of consistent polynomials in terms of which the multidimensionally consistent systems are defined, in particular providing an explanation of the spectral curve and the natural parameterisation of these systems in terms of points on that curve. The objective here is different, namely to understand the full array of possible singularities in a global sense.

The local analysis of ABS in [5] showed that singularities in the solutions of these equations are naturally associated with the edges of the lattice (specifically with the vanishing of edge biquadratics). This gives rise to the question of how to determine globally the collections of edges that are admissible singularities. There turn out to be a rich variety of possibilities, singularities can be isolated or they can form barriers partitioning the lattice, and their precise shape, or configuration, can vary a lot. The first main outcome of this work is a set of geometric criteria which provide a basic test for the admissibility of a singularity configuration.

The general theory of the ABS equations often relies on being able to apply the Bäcklund transformation to a given solution, or in other words to extend the solution into a new lattice direction. The second half of this paper (Section 3) will describe the obstruction singularities

* This paper is a contribution to the Proceedings of the Conference "Symmetries and Integrability of Difference Equations (SIDE-9)" (June 14-18, 2010, Varna, Bulgaria). The full collection is available at http://www.emis.de/journals/SIGMA/SIDE-9.html 
Table 1. Canonical forms for the type-Q polynomials. $\alpha, \beta$ are complex parameters, $\delta \in\{0,1\}$ and $k \in \mathbb{C} \backslash\{-1,0,1\}$ is the modulus of the Jacobi sn function appearing in Q4. The given function $f$ serves to parameterise a curve $\left(f^{\prime}\right)^{2}=r(f)$ defined in terms of a polynomial $r$, which loosely speaking serves to characterise the equation itself $[6,5]$.

\begin{tabular}{lll}
\hline & $\mathcal{P}(u, \tilde{u}, \hat{u}, \hat{\tilde{u}})$ & $f(\zeta)$ \\
\hline Q4 & $\operatorname{sn}(\alpha)(u \tilde{u}+\hat{u} \hat{\tilde{u}})-\operatorname{sn}(\beta)(u \hat{u}+\tilde{u} \hat{\tilde{u}})$ & $\sqrt{k} \operatorname{sn}(\zeta)$ \\
& $-\operatorname{sn}(\alpha-\beta)[\tilde{u} \hat{u}+u \tilde{\tilde{u}}-k \operatorname{sn}(\alpha) \operatorname{sn}(\beta)(1+u \tilde{u} \hat{u} \hat{\tilde{u}})]$ & \\
Q3 $^{\delta}$ & $\sinh (\alpha)(u \tilde{u}+\hat{u} \hat{\tilde{u}}-\sinh (\beta)(u \hat{u}+\tilde{u} \tilde{\tilde{u}})$ & \\
& $-\sinh (\alpha-\beta)\left(\tilde{u} \hat{u}+u \hat{\tilde{u}}+\delta^{2} \sinh (\alpha) \sinh (\beta)\right)$ & $\frac{1}{2}\left[\exp (\zeta)+\delta^{2} \exp (-\zeta)\right]$ \\
& $\alpha(u-\hat{u})(\tilde{u}-\hat{\tilde{u}})-\beta(u-\tilde{u})(\hat{u}-\hat{\tilde{u}})$ & \\
Q2 & $+\alpha \beta(\alpha-\beta)\left(u+\tilde{u}+\hat{u}+\hat{\tilde{u}}-\alpha^{2}+\alpha \beta-\beta^{2}\right)$ & $\zeta^{2}$ \\
Q1 $^{\delta}$ & $\alpha(u-\hat{u})(\tilde{u}-\hat{\tilde{u}})-\beta(u-\tilde{u})(\hat{u}-\hat{\tilde{u}})+\delta^{2} \alpha \beta(\alpha-\beta)$ & $\delta \zeta$ \\
\hline
\end{tabular}

can present to performing this operation. Demanding a solution be entirely free of singularities is a rather strong requirement and it is natural to expect that something weaker would be sufficient to guarantee the consistency of the Bäcklund equations, which is indeed the case. The main problematic singularities are those which are isolated, their effect is quantified by introducing a notion of monodromy. This can be used to resolve the global inconsistency of the Bäcklund equations, either by excluding from consideration those solutions whose singularities have non-trivial monodromy, or more interestingly through a relaxation of the definition of the domain of the transformed solution by allowing a modification of the lattice combinatorics.

The interaction between the Bäcklund transformation and singularities which form barriers is also of interest, in particular leading to a natural class of reductions of these systems. The main issues there are somewhat different and will therefore be the subject of a separate publication.

\section{The class of equations and singularities in the solutions}

The focus here will be on the equations of type-Q defined in [5]. This is a restricted set of the discrete $\mathrm{KdV}$-type equations, and this restriction requires some justification. Specifically, equations which are not of type-Q have the additional feature of singular values, special points in $\mathbb{C} \cup\{\infty\}$ (and usually canonical forms dictate these are 0 or $\infty$ ) whose presence in a solution indicates a singularity. The type-Q equations have no such special points, however it will be seen that they do have a rich theory of singularities, many aspects of which translate to the equations which are not of type-Q. The presence of singular values mean the non- $\mathrm{Q}$ (or type-H) equations deserve a separate treatment, the precise singularity structure being essentially different.

\subsection{Basic definitions}

The equations to be considered take the form

$$
\mathcal{P}(u, \tilde{u}, \hat{u}, \hat{\tilde{u}})=0
$$

where $\mathcal{P}$ is one of the polynomials listed in Table 1 . $u=u(n, m), \tilde{u}=u(n+1, m), \hat{u}=$ $u(n, m+1)$ and $\hat{\tilde{u}}=u(n+1, m+1)$ are values of the dependent variable $u$ as a function of independent variables $n, m \in \mathbb{Z}$, therefore equation (2.1) connects values of $u$ on the vertices of each quadrilateral of the $\mathbb{Z}^{2}$ lattice. Our intention is to describe singularities for all equations of the form (2.1) defined by type-Q polynomials with Kleinian symmetry, but according to [5] we can restrict attention to the canonical forms given in Table 1 without losing generality. 
Consider numbering the four vertices of a quadrilateral in the order the corresponding variables appear in the argument of $\mathcal{P}$ in (2.1). This allows the definition:

\section{Definition 1.}

(i) A solution of (2.1) is said to be singular with respect to vertex $i$ of some quadrilateral if

$$
\left[\partial_{i} \mathcal{P}\right](u, \tilde{u}, \hat{u}, \hat{\tilde{u}})=0
$$

also holds on that quadrilateral.

(ii) The set of all quadrilaterals on which the solution is singular is called the singular region.

Here $\partial_{i} \mathcal{P}$ denotes the partial derivative of $\mathcal{P}$ with respect to its $i^{\text {th }}$ argument, so actually has no dependence on the $i^{\text {th }}$ argument because $\mathcal{P}$ is a polynomial of degree one in each argument. This definition means a solution is singular with respect to vertex $i$ on some quadrilateral when it satisfies (2.1) independently of its value on vertex $i$.

As an explicit example consider the solution mentioned in the introduction which is singular everywhere. This solution is of the form

$$
u=f(\zeta)
$$

with $f$ written in Table 1 and $\zeta$ a function defined on $\mathbb{Z}^{2}$ by the system

$$
\tilde{\zeta}=\zeta+\epsilon_{1} \alpha, \quad \hat{\zeta}=\zeta+\epsilon_{2} \beta,
$$

where

$$
\epsilon_{1}, \epsilon_{2}: \mathbb{Z}^{2} \longrightarrow\{-1,+1\}, \quad \tilde{\epsilon}_{2}=\epsilon_{2}, \quad \hat{\epsilon}_{1}=\epsilon_{1} .
$$

The functions $\epsilon_{1}$ and $\epsilon_{2}$ are constant in one lattice direction so that the system (2.3) is compatible, and they attach an arbitrary +1 or -1 to the edges of the lattice in the other lattice direction. It can be directly verified that the resulting function $u$ defined in $(2.2)$ is a solution of the equation which is singular with respect to two diagonally opposite vertices on every quadrilateral (the relative signs of $\epsilon_{1}$ and $\epsilon_{2}$ determine which diagonal pair).

\subsection{Singular edges}

It can be seen from Definition 1 that to locate a singularity requires the specification of a quadrilateral, as well as a particular vertex of that quadrilateral. However the description of singularities can be simplified by considering the polynomials

$$
\mathcal{H}_{i j}=\left(\partial_{i} \mathcal{P}\right)\left(\partial_{j} \mathcal{P}\right)-\left(\partial_{i} \partial_{j} \mathcal{P}\right) \mathcal{P}, \quad i, j \in\{1,2,3,4\}, \quad i \neq j .
$$

Clearly $\mathcal{H}_{i j}$ is invariant under interchange of the indices $i$ and $j$, also it is not difficult to verify that $\mathcal{H}_{i j}$, considered as a polynomial in four variables, is degree zero in arguments $i$ and $j$ (has no dependence on argument $i$ or $j$ ). It follows from the invariance of $\mathcal{P}$ under Kleinian permutations of its arguments (this invariance is easily verified by glancing at Table 1) that there are really only three polynomials defined by (2.4), specifically

$$
\begin{aligned}
& h_{12}(x, y):=\mathcal{H}_{12}(\cdot, \cdot, x, y)=\mathcal{H}_{34}(x, y, \cdot, \cdot), \\
& h_{13}(x, y):=\mathcal{H}_{13}(\cdot, x, \cdot, y)=\mathcal{H}_{24}(x, \cdot, y, \cdot), \\
& h_{14}(x, y):=\mathcal{H}_{14}(\cdot, x, y, \cdot)=\mathcal{H}_{23}(x, \cdot, \cdot, y) .
\end{aligned}
$$


Table 2. Biquadratics $h_{12}$ obtained from the polynomials listed in Table 1 . The other biquadratics can be obtained from these by the equations $h_{13}=\left.h_{12}\right|_{\alpha \leftrightarrow \beta}$ and $h_{14}=\left.h_{12}\right|_{\alpha \rightarrow \beta-\alpha}$.

\begin{tabular}{ll}
\hline & $h_{12}(x, y)$ \\
\hline Q4 & $\operatorname{sn}(\beta) \operatorname{sn}(\alpha-\beta)\left[x^{2}+y^{2}-\left(1+x^{2} y^{2}\right) \operatorname{sn}^{2}(\alpha)-2 x y \operatorname{cn}(\alpha) \operatorname{dn}(\alpha)\right]$ \\
Q3 $^{\delta}$ & $\sinh (\beta) \sinh (\alpha-\beta)\left[\left(y-x e^{\alpha}\right)\left(y-x e^{-\alpha}\right)+\delta^{2} \sinh ^{2}(\alpha)\right]$ \\
Q2 $^{2}$ & $\beta(\alpha-\beta)\left[y^{2}+x^{2}-2 x y-2 \alpha^{2}(x+y)+\alpha^{4}\right]$ \\
Q1 $^{\delta}$ & $\beta(\alpha-\beta)(y-x-\delta \alpha)(y-x+\delta \alpha)$ \\
\hline
\end{tabular}

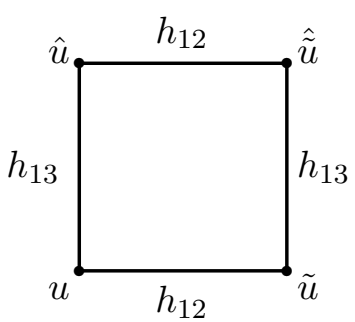

Figure 1. Values of the dependent variable associated to the vertices of a quadrilateral and biquadratics defined in (2.4), (2.5) associated to the edges.

These are the symmetric biquadratic polynomials which play a basic role in the theory of (2.1) developed in [6, 1, 5]. Explicit forms of the biquadratics are given in Table 2. The most important aspect here is that, excluding $Q 1^{0}$, these biquadratics are the addition formulae for the functions $f$ appearing in Table 1 , specifically

$$
\begin{array}{lll}
h_{12}(f(\zeta), f(\tilde{\zeta}))=0 & \Leftrightarrow & f(\tilde{\zeta})=f(\zeta \pm \alpha), \\
h_{13}(f(\zeta), f(\hat{\zeta}))=0 & \Leftrightarrow & f(\hat{\zeta})=f(\zeta \pm \beta), \\
h_{14}(f(\zeta), f(\hat{\tilde{\zeta}}))=0 & \Leftrightarrow & f(\hat{\tilde{\zeta}})=f(\zeta \pm \alpha \mp \beta) .
\end{array}
$$

From Definition $1(i)$ and the definition of $\mathcal{H}_{i j}$ in $(2.4)$ it can be seen that

Lemma 1 (ABS [5]). A solution of (2.1) satisfies $\mathcal{H}_{i j}(u, \tilde{u}, \hat{u}, \hat{\tilde{u}})=0$ on some quadrilateral if and only if it is singular with respect to vertex $i$ or $j$ of that quadrilateral.

This connection between singularities and the vanishing of the biquadratics is key in what follows.

Biquadratics $h_{12}$ and $h_{13}$ are naturally associated with the edges of the quadrilateral as shown in Fig. $1^{1}$. And because the biquadratics on opposite edges of the quadrilateral coincide, they can be associated in a consistent way to all edges of the lattice. The problem of describing singularities reduces to listing the edges of the lattice on which the biquadratics vanish, thus it is natural to make the following definition:

Definition 2. A solution of (2.1) is said to be singular on some edge of the lattice if the biquadratic on that edge (cf. Fig. 1) vanishes.

It then follows from Definition 1(ii) and Lemma 1 that

Lemma 2. A quadrilateral is part of the singular region (cf. Definition 1(ii)) if and only if it has a singular edge.

But not every collection of edges is an admissible singularity configuration.

\footnotetext{
${ }^{1}$ The remaining biquadratic $h_{14}$ is naturally associated with the diagonals.
} 
(a)

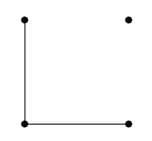

(b)
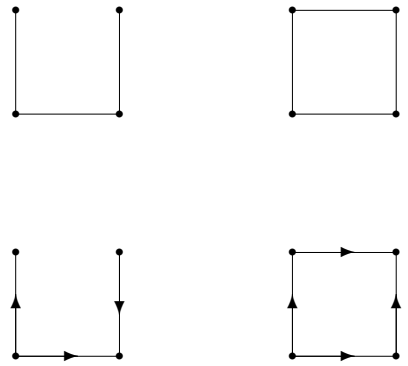

Figure 2. Admissible singularity configurations on a single quadrilateral in part (a), and attached arrows in part $(b)$. The solid lines indicate singular edges. The configurations are given up to the symmetries of the square and also up to an overall reversal of arrows in part $(b)$. In the exceptional case of equation $Q 1^{0}$ the second configuration involving three singular edges is inadmissible.

\subsection{Singularity configuration conditions}

Given a collection of edges we ask if they constitute an admissible singularity configuration. Constraints on admissible configurations can be broken down into the three conditions which follow. The first condition should be modified and the second removed altogether for equation $Q 1^{0}$, which is a special case whose solution is constant on vertices connected by singular edges.

The first condition concerns each quadrilateral within the singular region. It is clear from Lemma 1 that if a solution of (2.1) is singular on some edge of a quadrilateral, then it must also be singular on one of the two edges adjacent to the singular edge. This means that each quadrilateral within the singular region should be one of those shown in Fig. 2(a).

The second condition concerns each connected singular region. From Definition 2 and relations (2.6) the solution on a set of vertices connected by singular edges is of the form $u=f(\zeta)$ where $\tilde{\zeta}=\zeta+\epsilon_{1} \alpha, \hat{\zeta}=\zeta+\epsilon_{2} \beta$ and $\epsilon_{1}, \epsilon_{2}: \mathbb{Z}^{2} \rightarrow\{-1,+1\}$. The functions $\epsilon_{1}$ and $\epsilon_{2}$ attach a +1 or -1 to each singular edge, it is convenient to indicate this function geometrically as an arrow in the positive or negative lattice direction respectively. An analysis of (2.6) and Lemma 1 reveals that on each quadrilateral there is a constraint on these arrows, specifically they must be as shown in Fig. 2(b). Furthermore, it is necessary that around any closed path of singular edges the arrows sum to the null vector. This further condition is necessary for the compatibility of the aforementioned system for $\zeta$ and leads to explicit construction of the solution on the connected singular region.

Finally, supposing that the solution can be constructed on the singular region, we ask if a solution can then be constructed on the non-singular region. The only obstruction to this could be that the solution on this region is overdetermined. This can be checked at first by comparing the number of free vertices (i.e. vertices not attached to a singular edge) with the number of quadrilaterals (i.e. the number of constraints). If the solution on the non-singular region appears overdetermined by this test then further consideration is necessary because it might happen that some symmetry of the problem resolves the apparent inconsistency.

To summarise these conditions we formulate the following theorem.

Theorem 1. Propose a solution of (2.1) with some collection of singular edges (cf. Definition 2) which determine some singular region (cf. Lemma 2). Sufficient conditions for existence of this solution are as follows:

1. Each quadrilateral within the singular region should be one of those in Fig. 2(a).

2. It should be possible to attach arrows to all singular edges in such a way that $(i)$ each 
quadrilateral within the singular region is one of those shown in Fig. 2(b) and (ii) around any closed path of singular edges the arrows sum to the null vector ${ }^{2}$.

3. The solution on the non-singular region should not be overdetermined.

Singularity configurations which violate condition 2 are possible but are non-generic. Such configurations would require additional constraints connecting some or all of $\alpha, \beta$, the periodicity of $f$ (which are intrinsic to the equation) and the integration constant $\zeta_{0}$ on each connected singular region.

The conditions given in Theorem 1 make it possible to geometrically determine admissible singularity configurations, an example is illustrated in Fig. 3. Examples of (generically) inadmissible configurations which fail on conditions 2 and 3 respectively of Theorem 1 are shown in Fig. 4. It is necessary to account for the possibility of failure on condition 3 , however to conceive of an example took some time. In general a configuration which passes conditions 1 and 2 but which fails on condition 3 is rare, the example given on the right in Fig. 4 should be considered pathological.

There is an obvious algorithm to test these conditions, the algorithm would use a finite number of operations provided the singular region were finite. This is just a consequence of the equations being discrete; this determinism is not connected to the integrability. However, the Kleinian symmetry of the defining polynomials was essentially what led to the consistent association of singularities to edges of the lattice. This association is perhaps the most critical feature of the singularities because it simplifies many-fold their description. But the Kleinian symmetry is synonymous with the integrability for these systems, so the basic simplification of the problem afforded by association of singularities to edges is tied intimately to the integrability of the equations.

\subsection{Extension to quad-graphs and the multidimensional lattice}

This paper so-far has been focused on equations defined on the regular two-dimensional quadrilateral lattice, but the same equations can be considered in a more general setting. The restriction has been made in order to introduce the underlying ideas in as simple a setting as possible. In practice it is important to also consider the following two well-known generalisations.

First, the equations can be considered on the more general edge-labelled quad-graph as considered in [3]. It is natural to impose a mild technical assumption on the graph, which was established in [7], that characteristics do not self-intersect. This is because the system is basically unchanged by removing quadrilaterals on which the self-intersections occur.

Second, the equations can be considered on the regular multidimensional lattice. The extension of the underlying equation (2.1) to a system in $N$ dimensions is

$$
\mathcal{P}_{i j}\left(u, \mathrm{\top}_{i} u, \mathrm{\top}_{j} u, \mathrm{\top}_{i} \mathrm{\top}_{j} u\right)=0, \quad i, j \in\{1, \ldots, N\}, \quad i \neq j,
$$

where $\mathcal{P}_{i j}=\left.\mathcal{P}\right|_{(\alpha, \beta) \rightarrow\left(\alpha_{i}, \alpha_{j}\right)}, u$ takes values on the vertices of the $N$-dimensional regular lattice, $\mathrm{T}_{1}, \ldots, \mathrm{T}_{N}$ are shift operators in the $N$ dimensions, and $\alpha_{1}, \ldots, \alpha_{N}$ are a set of parameters ${ }^{3}$.

In the previous sections only the examples given relied on the setting of the regular twodimensional lattice, the definitions, lemmas and singularity configuration conditions have been stated in such a way that they are unchanged in the more general settings of quad-graph or regular multidimensional lattice. The most important fact that makes this possible is that the

\footnotetext{
${ }^{2}$ Condition 2 does not apply to equation $Q 1^{0}$.

${ }^{3}$ Actually, the generalisation to multidimensions contains the generalisation to quad-graphs because every quad-graph with non self-intersecting characteristics can be embedded in the regular multidimensional lattice [8]. Such embedding was used to establish the natural Cauchy-problem for the multidimensionally consistent equations on the quad-graph in [7].
} 

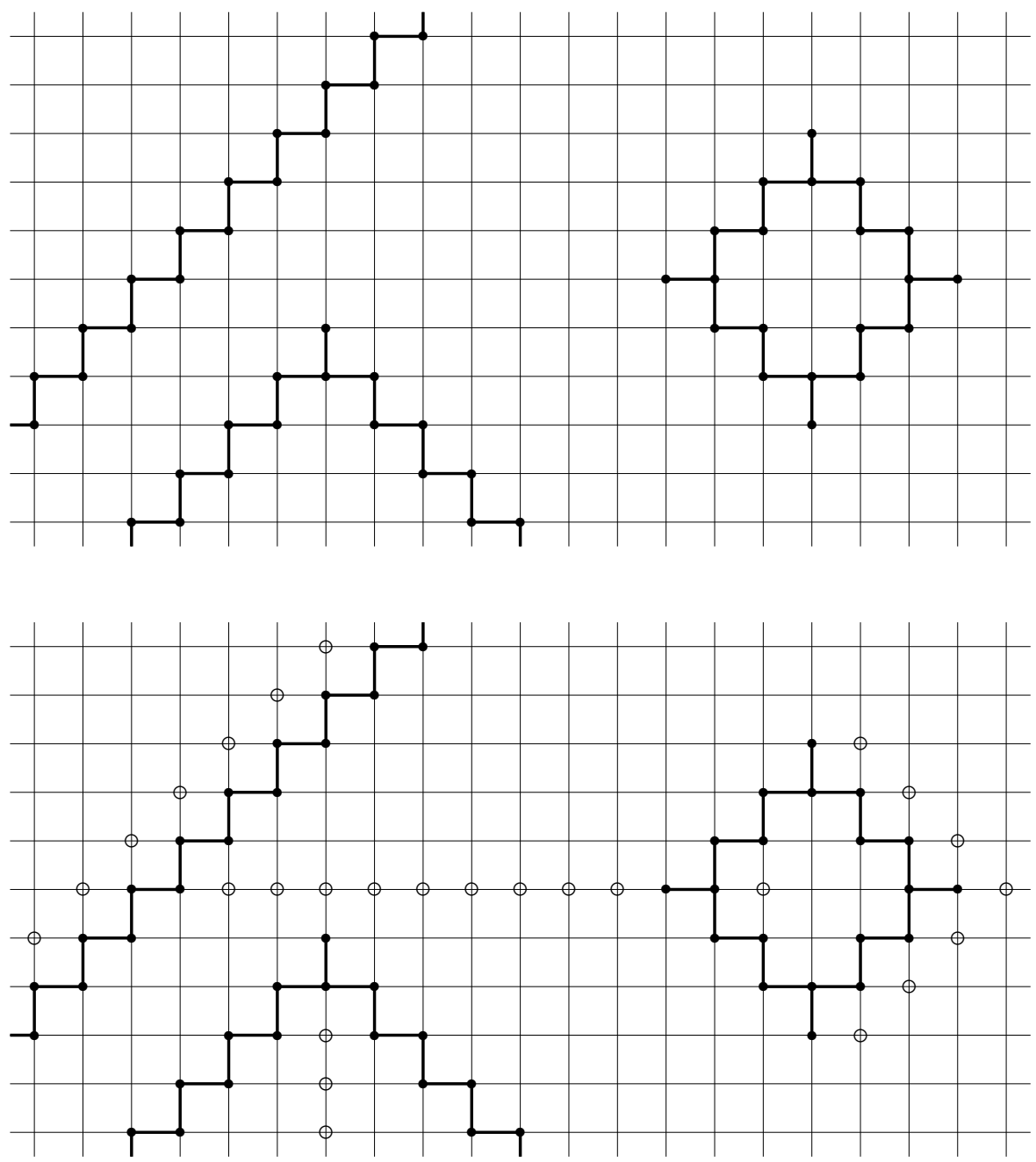

Figure 3. Example of an admissible configuration of singular edges in the solution of a type-Q lattice equation in two dimensions. The first diagram shows the singular edges which clearly satisfy condition 1 of Theorem 1. It is a simple exercise to attach arrows to the singular edges as described in condition 2. The second diagram is the same as the first but also shows an example set of vertices on which arbitrary non-singular initial data can be specified from which a unique solution is determined, thus demonstrating the configuration passes also condition 3 . Within the region completely enclosed by singularities there are five variables and four equations, so essentially one degree of freedom remains which is indicated by the single vertex on which initial data can be specified, actually there are still two solutions possible in this region so it is not quite determined uniquely here.

basic association of singularities to edges of the lattice continues to be valid. This relies on the result established in $[1,5]$ that the biquadratics on edges shared by any pair of quadrilaterals coincide up to a constant factor (this is not always true for consistent systems involving type- $\mathrm{H}$ polynomials). In conclusion, the singularity configuration conditions remain valid in both of these more general settings.

\section{Isolated singularities and monodromy}

The consideration of singularities in multidimensions (cf. Section 2.4) comes very close to fully describing the interaction between singularities and the Bäcklund transformation because ap- 

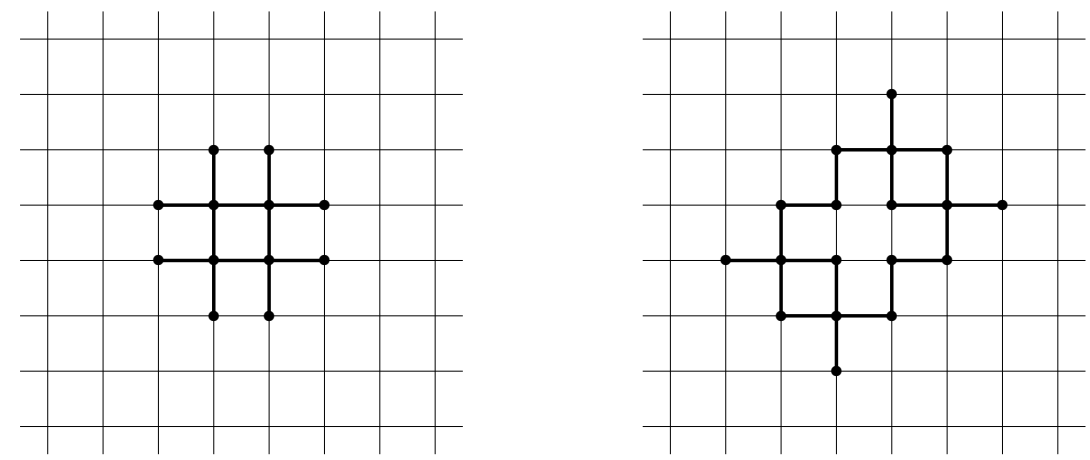

Figure 4. Collections of edges which fail the singularity configuration conditions of Theorem 1. It is easily checked that the first configuration passes condition 1 and $2(i)$ but fails on condition $2(i i)$, whilst the second configuration passes conditions 1 and 2 but a calculation shows that generically it fails on condition 3 .

plying the transformation means simply extending the solution one iterate into a new lattice direction. But there is one aspect of this interaction which that point of view overlooks. To describe this overlooked feature the question will be considered afresh, starting on the regular two-dimensional lattice (again, nothing important will be lost in this setting).

\subsection{The Bäcklund transformation}

The Bäcklund transformation provides a reason why it is natural to associate singularities to the edges of the lattice. The Bäcklund equations of (2.1) take the form

$$
\mathcal{B}_{1}(u, \tilde{u}, v, \tilde{v})=0, \quad \mathcal{B}_{2}(u, \hat{u}, v, \hat{v})=0,
$$

where $\mathcal{B}_{1}=\left.\mathcal{P}\right|_{(\alpha, \beta) \rightarrow(\alpha, \gamma)}$ and $\mathcal{B}_{2}=\left.\mathcal{P}\right|_{(\alpha, \beta) \rightarrow(\beta, \gamma)}(\mathcal{P}$ was the polynomial appearing in the equation (2.1) itself), they determine a new solution $v$ of (2.1) from an old solution $u$, the free parameter $\gamma$ is the Bäcklund parameter. Because $\mathcal{B}_{1}$ and $\mathcal{B}_{2}$ are polynomials of degree one the system (3.1) defines Möbius transformations $v \mapsto \tilde{v}$ and $v \mapsto \hat{v}$ associated with the (oriented) edges of the lattice. It happens that $u$ is singular on some edge exactly when the Möbius transformation is not defined on that edge. This occurs when (3.1) are reducible as polynomials in $(v, \tilde{v})$ and $(v, \hat{v})$ respectively.

\subsection{Monodromy}

The global compatibility of (3.1) as a system for $v$ means that the composition of Möbius transformations along some path connecting two vertices of the lattice should be independent of the path chosen. Now, the path-independence of composition around a single quadrilateral is equivalent to consistency of the polynomials on the cube. Path-independence of the composition in a global sense then follows when any path between two vertices can be deformed into any other path by changing it gradually on one quadrilateral at a time. This argument for global compatibility breaks down in the presence of isolated singularities, i.e. localised collections of edges on which the Möbius transformations are not defined, because if the two paths enclose a singularity they cannot be deformed one into the other in this way.

The possibility of an isolated singularity to result in global inconsistency of the Bäcklund system (3.1) can be quantified by introducing the monodromy of the singularity. Consider composing transformations along a closed path of non-singular edges enclosing the singularity. 
It is easily shown that deforming the path (without moving the point of origin) on one nonsingular quadrilateral at a time leaves the composed transformation unchanged. Whilst moving the point of origin or reversing the orientation of the path leads to conjugation of the composed transformation ${ }^{4}$. Up to conjugacy this composed transformation is the natural monodromy of the singularity:

\section{Definition 3.}

(i) An isolated singularity in a solution of (2.1) is a collection of singular edges for which there exists a finite-length enclosing path of non-singular edges.

(ii) Let $\mathrm{m}_{1}, \ldots, \mathrm{m}_{N}$ denote the Möbius transformations associated by the Bäcklund equations (3.1) to edges along a non-singular oriented path enclosing an isolated singularity such that the indices increase with distance along the path. Then the monodromy of the singularity is defined as the conjugacy class of the Möbius transformation $\mathrm{m}=\mathrm{m}_{N} \cdot \mathrm{m}_{N-1} \cdots \mathrm{m}_{1}$.

(iii) If this transformation is the identity we say the monodromy is trivial.

The monodromy is independent of the shape and orientation of the path. If it is trivial the singularity does not lead to global inconsistency of the Bäcklund system. It can be seen from Definition 3 and the explicit forms of the polynomials in Table 1 that the trivial-monodromy condition is polynomial in $f(\gamma)$ and $f^{\prime}(\gamma)$, where $\gamma$ is the Bäcklund parameter.

We remark that generically the potential for global inconsistency is always present for Bäcklund transformations defined by the polynomials consistent on a cube. It happens whenever the Bäcklund equations fail to define the Möbius transformations on some collection of edges, and therefore the monodromy can be defined in the same way for any similar Bäcklund transformations, including those involving type-H polynomials. There is, in principle, the possibility that singularities of the Bäcklund equations are distinct from singularities of the equation, this would indicate a kind of incompatibility between the singularity structure and the integrability, and it would be interesting to investigate to see if such systems exist (for instance amongst those listed in [9]) and to find out how the incompatibility is resolved.

\subsection{Calculation of monodromy}

To calculate the monodromy it is useful to exploit that there is a single complex parameter which distinguishes the conjugacy classes in the Möbius group. Consider the non-identity Möbius transformation $\mathrm{m}=x \mapsto(a x+b) /(c x+d)$, where $a, b, c, d \in \mathbb{C}, a d \neq b c$. Then the relationship between $\mathrm{m}$ and the complex parameter $\rho$

$$
\frac{(\rho+1)^{2}}{\rho}=\frac{\left(x-\mathrm{m}^{2}(x)\right)\left(\mathrm{m}(x)-\mathrm{m}^{3}(x)\right)}{(x-\mathrm{m}(x))\left(\mathrm{m}^{2}(x)-\mathrm{m}^{3}(x)\right)}=\frac{(a+d)^{2}}{a d-b c},
$$

where the second expression is independent of the arbitrary variable $x$, has the following properties. First, if $N \in \mathbb{Z}$ then $\mathrm{m} \rightarrow \mathrm{m}^{N} \Rightarrow \rho \rightarrow \rho^{N}$. Second, if $\mathrm{m}_{*}$ and $\rho_{*}$ are some other Möbius transformation and parameter related by (3.2), then $\left\{\rho_{*}, 1 / \rho_{*}\right\}=\{\rho, 1 / \rho\}$ if and only if $\mathrm{m}_{*}$ is conjugate to $\mathrm{m}$. These properties can be verified directly, or alternatively they can be deduced from matrix properties by observing that $\rho$ is the ratio of the eigenvalues of any matrix in $P G L(2, \mathbb{C})$ corresponding to the Möbius transformation $\mathrm{m}$ by the natural homomorphism. The expression (3.2) determines $\rho$ up to the transformation $\rho \rightarrow 1 / \rho$, which we identify with reversing the orientation of the path. This non-uniqueness allows $\rho$ to also encode the relative orientation of such paths, i.e., if one path enclosing a singularity has associated parameter $\rho$,

\footnotetext{
${ }^{4}$ The path-reversal of course leads to inversion of the transformation, but every Möbius transformation is conjugate to its inverse.
} 

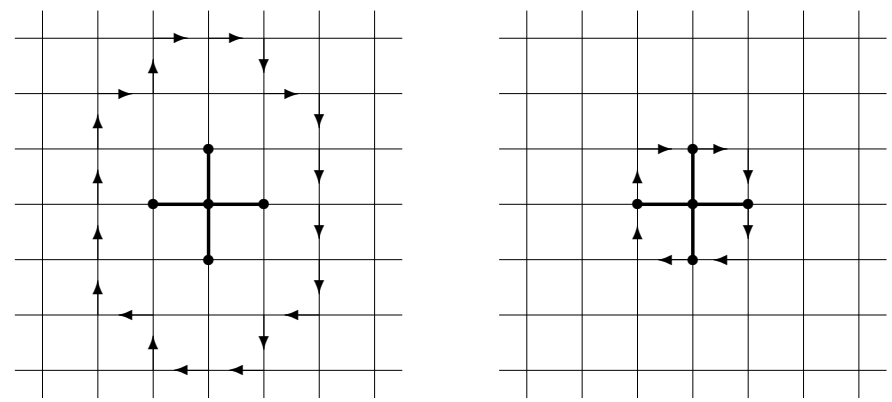

Figure 5. The simplest admissible singularity configuration. The first diagram shows a non-singular path enclosing the singularity. The shortest such path is indicated in the second diagram. The paths can be deformed into each other by making alterations on a single quadrilateral at a time whilst avoiding singular edges.

then a path with the opposite orientation enclosing the same singularity is naturally assigned the parameter $1 / \rho$. Thus the single complex parameter $\rho$ captures perfectly the monodromy of an isolated singularity.

\subsection{Explicit example of non-trivial monodromy}

An explicit example will now be used to demonstrate that it is quite normal for isolated singularities to have non-trivial monodromy, i.e. that isolated singularities will generally lead to the Bäcklund system (3.1) being inconsistent globally despite its local consistency. Furthermore, examining this example will also suggest an alternative and perhaps more intuitive characterisation of this situation.

To exhibit non-trivial monodromy it is sufficient to consider the simplest isolated singularity, which is the configuration of four edges in the form of a cross illustrated in Fig. 5. Two nonsingular paths enclosing the singularity are also shown. Clearly it is convenient to consider the shortest enclosing path shown on the right; to calculate explicitly the monodromy using this path is a straightforward but quite lengthy calculation. If the values of the function $u$ on the nine points of the singular region are represented by the following matrix

$$
\left[\begin{array}{ccc}
\hat{\hat{u}} & \hat{\tilde{u}} & \hat{\tilde{\tilde{u}}} \\
\hat{u} & \hat{\tilde{u}} & \hat{\tilde{\tilde{u}}} \\
u & \tilde{u} & \tilde{\tilde{u}}
\end{array}\right],
$$

then a solution of (2.1) with the indicated singularity in this region is represented by

$$
\left[\begin{array}{ccc}
c & f\left(\zeta_{0}+\beta\right) & d \\
f\left(\zeta_{0}+\alpha\right) & f\left(\zeta_{0}\right) & f\left(\zeta_{0}+\alpha\right) \\
a & f\left(\zeta_{0}+\beta\right) & b
\end{array}\right]
$$

Here the function $f$ depends on which type-Q polynomial is being considered, this function was given in the right-hand column of Table 1. It can be verified directly that (3.3) satisfies (2.1) on each of the four quadrilaterals independently of the data

$$
\left\{a, b, c, d, \zeta_{0}\right\}
$$

which is therefore arbitrary. Composing the eight transformations around the enclosing path results in a monodromy transformation which is the identity if and only if the following condition 
is satisfied by this data:

$$
\begin{aligned}
& {[a+d-b-c]\left[a d-f\left(\zeta_{0}+\alpha-\beta\right) f\left(\zeta_{0}-\alpha+\beta\right)\right]} \\
& \quad=[a d-b c]\left[a+d-f\left(\zeta_{0}+\alpha-\beta\right)-f\left(\zeta_{0}-\alpha+\beta\right)\right] .
\end{aligned}
$$

Therefore for generic data the singularity in Fig. 5 has non-trivial monodromy. Notice that condition (3.4) is independent of the Bäcklund parameter $\gamma$, in fact the singularity in Fig. 5 belongs to an exceptional sub-class of configurations whose monodromy turns out to be independent of this parameter.

The condition (3.4) itself is quite interesting, in fact this condition is nothing but the discrete Toda system $[1,10]$ which in the absence of singularities is a consequence of the equations on the quadrilaterals. Therefore in this example there is a direct connection between asking if the solution satisfies the larger-stencil formal consequences of (2.1) and whether there is a singularity with non-trivial monodromy.

\subsection{Formal consequences of the equation}

To better understand the connection observed in the previous section it is useful to focus on the path which forms the external boundary of the singular region. It is a corollary of Lemma 1 that the following holds:

Lemma 3. Edges which lie on the boundary of the singular region are themselves non-singular.

For instance the path indicated on the right of Fig. 5 traverses the boundary of the singular region. Clearly the monodromy of an isolated singularity can be computed by composing transformations along its external boundary. Therefore the monodromy must only depend on the values of the solution on this boundary. This leads to the following:

Proposition 1. Consider an isolated singularity in a solution of (2.1). The constraints on the values of the solution on the external boundary of the singular region which arise when (2.1) is used to eliminate variables on vertices interior to this boundary are sufficient for the monodromy to be trivial.

The sufficiency becomes clear by considering the same path, but with the singularity removed: in the absence of the singularity the formal constraints arising on the external boundary must be satisfied, and the monodromy is of course trivial. The trivial monodromy must therefore be a consequence of these constraints as the monodromy itself depends only on the values of the solution on the external boundary.

It would be natural to conjecture that the sufficiency described in Proposition 1 can be strengthened to sufficiency and necessity. It would require a more precise formulation, in particular demanding that the monodromy vanishes for all values of the spectral parameter. Such a proposition can in fact be proven for simple configurations, however counter-examples do exist in the case when the underlying system of polynomials are more degenerate than the type- $\mathrm{Q}$ systems considered here, thus any proof will rely on further analytic considerations of the polynomials and goes beyond the scope of the present paper.

Suffice it to say that imposing not just the quadrilateral equation (2.1), but also the largerstencil formal consequences of the equation, restricts solutions to those whose singularities have trivial monodromy.

\subsection{Smooth deformation of a non-singular solution}

There is a second point of view which in addition to Proposition 1 provides a different but similarly intuitive way to understand of the difference between singularities with trivial and non-trivial monodromy. It can be stated thus: 

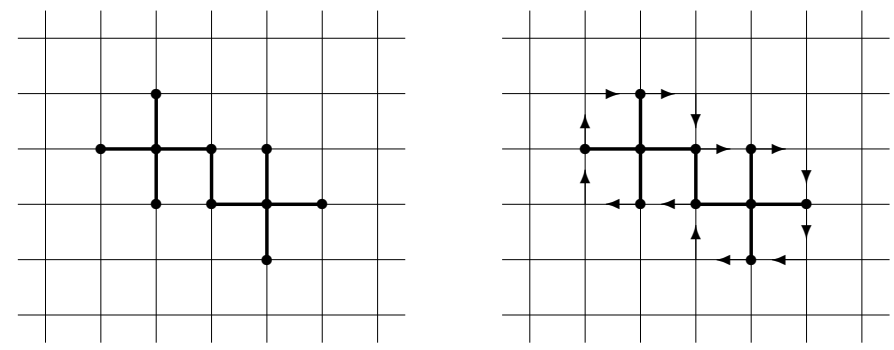

Figure 6. An admissible singularity configuration which has non-trivial monodromy by virtue of its shape alone. The second diagram is the same as the first but includes a path traversing the boundary of the singular region.

Proposition 2. If a solution of (2.1) has been obtained from a non-singular solution by a smooth limiting procedure, then any singularities present must have trivial monodromy.

The proof of this statement is very simple. The monodromy around any path is of course trivial for the non-singular solution. This remains true as the solution is deformed to approach the solution containing singularities. In the limit the monodromy around any non-singular path must remain trivial because it is a smooth function of the values of the solution which are themselves being smoothly deformed.

Again, in the consideration of isolated singularities, it is natural to conjecture the converse of Proposition 2, but obtaining a proof is equal in complexity to proving the converse of Proposition 1 and is beyond the scope of the present paper.

The Propositions 1 and 2 give alternative (but clearly connected) ways to understand the difference between isolated singularities which have trivial and non-trivial monodromy. The reason these concepts are important is that, unlike the monodromy, they can be understood without first knowing about the integrability.

\subsection{The Bäcklund transformation and non-trivial lattice combinatorics}

To apply the Bäcklund transformation it is quite natural to restrict attention to solutions whose singularities have trivial monodromy because the transformed solution is then well defined on $\mathbb{Z}^{2}$. In this respect it is worth remarking that demanding an isolated singularity have trivial monodromy (for all values of the Bäcklund parameter) imposes conditions on its shape in addition to those of Section 2.3. In other words there exist admissible singularity configurations which necessarily have non-trivial monodromy (for a generic value of the Bäcklund parameter). Such a configuration is illustrated in Fig. 6. What happens in this example is that imposing the trivial-monodromy conditions on the boundary forces some of the boundary edges to become singular, making the singular region change shape.

However it is not necessary to restrict attention only to solutions whose singularities have trivial monodromy. Whilst the transformed solution may not be well defined on the $\mathbb{Z}^{2}$ lattice, it is well defined on a lattice with different combinatorics. An example of this can be exhibited by returning to the configuration shown in Fig. 5. We suppose the monodromy is non-trivial, but will consider the simplest non-trivial situation which occurs when the square of the monodromy transformation is the identity, in particular this means that $\rho=-1$. The transformed solution is not defined on $\mathbb{Z}^{2}$, the actual lattice on which it is uniquely defined is illustrated in Fig. 7. This lattice can be viewed as two copies of $\mathbb{Z}^{2}$ with the vertex at the centre of the singularity removed, and which are then joined together along a branch cut along a path connecting the removed vertex with a point at infinity. The lattices should be joined in such a way that a path once around the removed vertex causes a switch from one lattice onto the other. The new lattice 


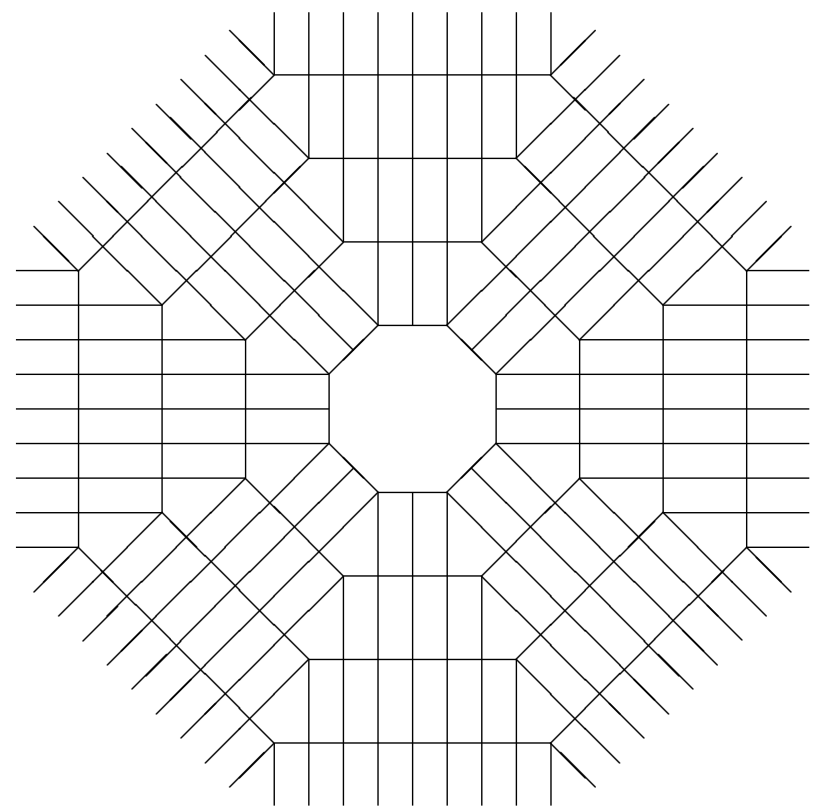

Figure 7. A quadrilateral lattice with combinatorics distinct from $\mathbb{Z}^{2}$. It is the lattice on which the transformed solution is defined if the Bäcklund transformation is applied to a solution with the singularity configuration of Fig. 5 in the case that the square of the monodromy transformation is the identity.

could be visualised as the embedding of a two-dimensional lattice in three dimensions, Fig. 7 gives an alternative way to view the lattice - the combinatorics are different but it is embedded in just two dimensions.

Thus it is natural that in the presence of singularities the Bäcklund transformation connects solutions of the same equation, but on domains with different underlying combinatorics. The precise combinatorics are a function of the singularity configuration and the monodromy. The procedure of defining the domain of the transformed solution is analogous to analytic continuation.

In conclusion, the restriction to solutions whose singularities have trivial monodromy means the Bäcklund transformation can be considered as usual: one iterate into a new direction on the regular multidimensional lattice. Lifting this restriction leads to an interesting situation that can also be described on a higher dimensional lattice, but with the difference that the combinatorics of the lattice will depend on the position, shape and monodromy of the singularities.

\subsection{Remark on the monodromy in multidimensions}

The important generalisation to multidimensions requires a fresh look at the notion of monodromy. We start with the Bäcklund transformation in multidimensions; given a solution $u$ of the $N$-dimensional system (2.7) the Bäcklund equations for a new solution $v$ are

$$
\mathcal{B}_{i}\left(u, \mathrm{~T}_{i} u, v, \mathrm{~T}_{i} v\right)=0, \quad i \in\{1, \ldots, N\},
$$

where $\mathcal{B}_{i}=\left.\mathcal{P}\right|_{(\alpha, \beta) \rightarrow\left(\alpha_{i}, \gamma\right)}$. The relationship between the Bäcklund transformation and the singular edges is a direct extension of the two-dimensional situation: the equations (3.5) associate Möbius transformations $v \mapsto \mathrm{T}_{i} v$ to all edges of the multidimensional lattice except those on which solution $u$ is singular.

To see how the idea of monodromy extends to situations beyond $N=2$ suppose first that $N=3$. It is clear that isolated singularities in three dimensions do not lead to global inconsistency of (3.5) because they make no obstruction to gradual deformation of one lattice path 
into another. The singularities which cause obstruction to deformations of this kind in three dimensions include singularities which form a closed loop, or which are infinite in extent in one dimension. That such singularities could have non-trivial monodromy relies on the fact pointed out in Section 3.2: the trivial monodromy at a fixed value of the spectral parameter which is necessary for the extension of a two-dimensional solution to three dimensions, does not mean the monodromy is trivial for all values of the parameter.

In general then, the class of singularities with the potential to have non-trivial monodromy needs to be considered in the context of the dimension of the underlying system. Only in two dimensions are the singularities with non-trivial monodromy necessarily isolated.

\section{Acknowledgements}

The author gratefully acknowledges helpful discussions with Nalini Joshi. The research was funded by Australian Research Council Discovery Grants DP 0985615 and DP 110104151.

\section{References}

[1] Adler V.E., Bobenko A.I., Suris Yu.B., Classification of integrable equations on quad-graphs. The consistency approach, Comm. Math. Phys. 233 (2003), 513-543, nlin.SI/0202024.

[2] Nijhoff F.W., Walker A.J., The discrete and continuous Painlevé VI hierarchy and the Garnier systems, Glasg. Math. J. 43A (2001), 109-123, nlin.SI/0001054.

[3] Bobenko A.I., Suris Yu.B., Integrable systems on quad-graphs, Int. Math. Res. Not. 2002 (2002), no. 11, 573-611, nlin.SI/0110004.

[4] Atkinson J., Hietarinta J., Nijhoff F.W., Seed and soliton solutions for Adler's lattice equation, J. Phys. A: Math. Theor. 40 (2007), F1-F8, nlin.SI/0609044.

[5] Adler V.E., Bobenko A.I., Suris Yu.B., Discrete nonlinear hyperbolic equations: classification of integrable cases, Funct. Anal. Appl. 43 (2009), 3-17, arXiv:0705.1663.

[6] Adler V.E., Bäcklund transformation for the Krichever-Novikov equation, Int. Math. Res. Not. 1998 (1998), no. 1, 1-4, solv-int/9707015.

[7] Adler V.E., Veselov A.P., Cauchy problem for integrable discrete equations on quad-graphs, Acta Appl. Math. 84 (2004), 237-262, math-ph/0211054.

[8] Dolbilin N.P., Sedrakyan A.G., Shtan'ko M.A., Shtogrin M.I., Topology of a family of parametrizations of two-dimensional cycles arising in the three-dimensional Ising model, Dokl. Akad. Nauk SSSR 295 (1987), no. 1, 19-23 (English transl.: Soviet Math. Dokl. 36 (1988), no. 1, 11-15).

[9] Boll R., Classification of 3D consistent quad-equations, arXiv:1009.4007.

[10] Adler V.E., Suris Yu.B., Q4: integrable master equation related to an elliptic curve, Int. Math. Res. Not. 2004 (2004), no. 47, 2523-2553, nlin.SI/0309030. 